\title{
Effects of Respiratory-Gated Auricular Vagal Nerve Stimulation (RAVANS) on Nonlinear Heartbeat Dynamics in Hypertensive Patients
}

\author{
Ronald G. Garcia ${ }^{*}, 1,2$, Roberta Sclocco*,1,3, Aileen Gabriel ${ }^{2}$, Gaetano Valenza $^{4}$, \\ Vitaly Napadow ${ }^{1,2}$, Riccardo Barbieri ${ }^{1,5}$ \\ *authors contributed equally to this manuscript \\ ${ }^{1}$ Massachusetts General Hospital, Harvard Medical School, Boston, MA, USA \\ ${ }^{2}$ Brigham and Women's Hospital, Harvard Medical School, Boston, MA, USA \\ ${ }^{3}$ Logan University, Chesterfield, MO, USA \\ ${ }^{4}$ Research Center E. Piaggio, University of Pisa, Pisa, Italy \\ ${ }^{5}$ Department of Electronics, Information and Bioengineering, Politecnico di Milano, Milan, Italy
}

\begin{abstract}
The association between hypertension and cardiac autonomic dysfunction is well recognized and has been linked to the progression of coronary heart disease and heart failure, suggesting that autonomic control pathways may be a therapeutic target. In this study, we investigate the effects of a novel, respiratory-gated, auricular vagal afferent nerve stimulation (RAVANS) technique on heartbeat dynamics of hypertensive patients. Twelve hypertensive subjects underwent two experimental sessions on non-consecutive days. In each session subjects performed an initial paced-breathing (PB) task without intervention, and a second $P B$ task with either RAVANS or sham stimulation. Electrocardiogram and respiration signals were collected and point process nonlinear analysis of heartbeat dynamics was performed to obtain instantaneous time-domain $\left(\mu_{R R}, \sigma_{R R}\right)$, spectral $(L F, H F, L F / H F)$ and bispectral $(L L, L H$, and $H H)$ features. We found that exhalatory-gated RAVANS resulted in a significant increase in $\mu_{R R}, H F$ and $H H$ compared with $P B$ alone and sham, revealing modulatory effects of this technique on complex dynamics associated with parasympathetic activity. We conclude that exhalatory-gated RAVANS could be a promising intervention for the treatment of cardiac autonomic dysfunction in hypertensive subjects.
\end{abstract}

\section{Introduction}

Hypertension is associated with alterations in cardiac autonomic modulation characterized by an increased sympathetic activity and a reduced vagal tone [1]. This is of particular importance given that reduced vagal activity is a predictor of cardiovascular morbidity and mortality even in low risk populations [2], which suggests this marker as a potential therapeutic target in hypertensive subjects.

Previous experimental and clinical reports have shown that Vagus Nerve Stimulation (VNS) could have beneficial effects on the modulation of cardiac autonomic activity [3]. However, some disadvantages of VNS such as the risks of operation, including lesions of the vagus nerve, infection, hoarseness, and shortness of breath limits its applicability [4]. In the last decade, an alternative transcutaneous method of stimulating afferent terminals of the vagus nerve was proposed [5]. This method involves the electrical stimulation of the auricular afferent branch of the vagus nerve (ABVN). Previous studies have suggested that transcutaneous VNS (t-VNS) could increase vagal tone in healthy subjects [6], however others reports have not evidenced significant effects of tVNS in heart rate or heart rate variability (HRV) [7]. One possible explanation for these conflicting results could be the limitations of traditional HRV analyses methods when a high resolution in the time and frequency domains is needed. In addition, no studies have been performed to evaluate the therapeutic potential of t-VNS in reducing the cardiac autonomic imbalance in subjects with hypertension. Moreover, as autonomic nuclei that receive vagal afference in the medulla operate in tune with respiration [8], our group has proposed that t-VNS effects in cardiac autonomic modulation may be optimized by gating the electrical stimulation to specific phases of the respiratory cycle.

Here, we evaluate the effects of respiratory-gated auricular vagal afferent nerve stimulation (RAVANS) in the modulation of heartbeat dynamics in hypertensive subjects. To this end, we employ a nonlinear point- 
process framework [9, 10], looking for significant differences between hypertensive subjects randomized to sham vs active stimulation using HRV metrics defined in the time and frequency domains, as well as features defined in the bispectral plane.

\section{Materials and methods}

\subsection{Study population}

Twelve hypertensive subjects $(53.2 \pm 5.8$ years, 7 males) were enrolled in the study. All subjects were on stable doses of antihypertensive medications at least 30 days prior to entering the study. Exclusion criteria were history of other known cardio-, cerebro-, or peripheral vascular disease, presence of neoplasm, diabetes mellitus, kidney or liver failure, infectious or systemic inflammatory disease and current neurological illness. All subjects gave written informed consent approved by an Institutional Review Board.

\subsection{Experimental protocol}

Participants underwent 2 sessions on non-consecutive days. In each session, they were asked to perform two non-consecutive 2-minute paced breathing (PB) tasks, and RAVANS was delivered during the second one (RAVANS+PB). The paced respiratory cycle had a $5 \mathrm{~s}$ duration ( $2.5 \mathrm{~s}$ inhalation, $2.5 \mathrm{~s}$ exhalation), in order to be in the physiological range of respiration frequency at rest. Using a fixed respiratory cycle also allowed us to control for the amount of stimulation received by each subject.

Subjects were randomly assigned to receive active or sham RAVANS. Active RAVANS was delivered at 25 $\mathrm{Hz}$ (duration:1s, pulse width: $15 \mathrm{~ms}$ ) and gated to exhalation, with current intensity set to achieve moderate (but not painful) sensation. Sham stimulation was delivered with the current generator shut off, with subjects blinded to stimulus condition. Continuous ECG (lead II) was collected at $400 \mathrm{~Hz}$ using a using Chart Data Acquisition Software (ADInstruments, Colorado Springs, CO) on a laptop equipped with a 16-channel Powerlab DAQ System (ADInstruments).

\subsection{Point-process model of the heartbeat}

R-wave peaks from ECG signals were automatically detected using in-house algorithms, and then visually inspected in order to ensure correct peak identification. By using the inhomogeneous point-process framework [9, $10]$, we model the unevenly sampled RR interval series through probability density functions $(p d f)$ characterizing the time until the next event occurs as a function of the past history. Within this framework, Laguerre expansions of the Wiener-Volterra linear and nonlinear autoregressive terms account for long-term nonlinear information [10]. As major advantages, instantaneous measures can be estimated without applying any interpolation techniques to the RR interval series, and are associated to effective goodness-of-fit measures.

Mathematically, the $p d f$ characterizing the time of the next ventricular contraction is an inverse Gaussian function:

$f\left(t \mid \mathcal{H}_{t}, \xi(t)\right) \sqrt{\frac{\xi_{0}(t)}{2 \pi\left(t-u_{j}\right)^{3}}} \exp \left\{-\frac{1}{2} \frac{\xi_{0}(t)\left[t-u_{j}-\mu_{\mathrm{RR}}\left(t, \mathcal{H}_{t}, \xi(t)\right)\right]^{2}}{\mu_{\mathrm{RR}}\left(t, \mathcal{H}_{t}, \xi(t)\right)^{2}\left(t-u_{j}\right)}\right\}$

where: ; $\left\{u_{j}\right\}_{j=1}^{J}$ are R-wave events, $\mathrm{RR}_{j}=u_{j}-u_{j-1}>0$ the $j^{\text {th }} \mathrm{RR}$ interval; $\mathcal{H}_{t}=\left(u_{j}, \mathrm{RR}_{j}, \mathrm{RR}_{j-1}, \ldots, \mathrm{RR}_{j-M+1}\right)$; $\xi(t)$ is the vector of the model time-varying parameters; $\mu_{\mathrm{RR}}\left(t, \mathcal{H}_{t}, \xi(t)\right)$ is the mean of the distribution; and $\xi_{0}(t)>0$ is the shape parameter of the inverse Gaussian distribution.

$\mathrm{Eq}(1)$ is parametrized in its first-order moment as a Nonlinear Autoregressive model with Laguerre expansions (NARL) of the Volterra terms:

$$
\begin{gathered}
\mu_{\mathrm{RR}}\left(t, \mathcal{H}_{t}, \xi(t)\right)=\mathrm{RR}_{\widetilde{N}(t)}+g_{0}(t)+\sum_{i=0}^{p} g_{1}(i, t) l_{i}\left(t^{-}\right) \\
+\sum_{i=0}^{q} \sum_{j=0}^{q} g_{2}(i, j, t) l_{i}\left(t^{-}\right) l_{j}\left(t^{-}\right)
\end{gathered}
$$

where

$$
l_{i}\left(t^{-}\right)=\sum_{n=1}^{\widetilde{N}(t)} \phi_{i}(n)\left(\operatorname{RR}_{\widetilde{N}(t)-n}-\mathrm{RR}_{\widetilde{N}(t)-n-1}\right)
$$

is the output of the Laguerre filters just before time $t$, and $\phi_{i}(n)=$

$$
=\alpha^{\frac{n-i}{2}}(1-\alpha)^{\frac{1}{2}} \sum_{k=0}^{i}(-1)^{k}\left(\begin{array}{l}
n \\
k
\end{array}\right)\left(\begin{array}{l}
i \\
k
\end{array}\right) \alpha^{i-k}(1-\alpha)^{k}
$$

is the $i^{\text {th }}$-order discrete time orthonormal Laguerre function and $\alpha$ is the discrete-time Laguerre parameter $(0<\alpha<1)$ which determines the rate of exponential asymptotic decline.

The Laguerre expansion on the derivative R-R series improves stationarity within the sliding time window $W$ (in this work we have chosen $W=90 \mathrm{~s}$ ) [10].

As $\mu_{\mathrm{RR}}\left(t, \mathcal{H}_{t}, \xi(t)\right)$ is defined in continuous time, it is possible to obtain an instantaneous $\mathrm{R}-\mathrm{R}$ mean estimate at arbitraty timescales without interpolating between the arrival times of two consecutive heartbeats.

Concerning parameter estimation, we initialize the Newton-Raphson procedure at $t$ with the previous local maximum-likelihood estimate at time $t-\Delta$, where $\Delta$ defines the time interval shift to compute the next parameter update. We determine the optimal order $\{p, q\}$ based on the Kolmogorov-Smirnov (KS) test and associated KS statistics [10]. Autocorrelation plots are also visually inspected to ensure that all points of the plot are within the $95 \%$ of the confidence interval, hence guaranteeing the independence of the model-transformed intervals [10]. 


\subsection{Instantaneous time, frequency, and higher-order spectral analysis}

The time-domain characterization is based on the first and the second order moments of the underlying probability structure. Given the time-varying parameter set $\xi(t)$, the instantaneous mean $\mu_{R R}$, and R-R interval standard deviation $\sigma_{R R}$ can be extracted at each moment in time [10]. Estimates in the frequency domain are obtained by integrating the instantaneous autospectrum [10] within low frequency $(L F=0.05-0.15 \mathrm{~Hz})$, and high frequency $(\mathrm{HF}=0.15-0.5 \mathrm{~Hz})$ ranges, along with their ratio $(\mathrm{LF} / \mathrm{HF})$.

The higher-order spectral representation allows for the estimation of statistics beyond the second order, and phase relations between frequency components which would otherwise be suppressed. A detailed description of the instantaneous bispectrum $\left(B\left(f_{1}, f_{2}, t\right)\right)$ derivation from point-process nonlinear models can be found in [10]. These measures allow to evaluate the instantaneous presence of nonlinearity in heartbeat series by calculating nonlinear sympatho-vagal interactions. Specifically, by integrating the estimated bispectrum in the appropriate frequency bands, it is possible to obtain:

$$
\begin{aligned}
& \mathrm{LL}(t)=\int_{f_{1}=0^{+}}^{0.15} \int_{f_{2}=0^{+}}^{0.15} \mathrm{~B}\left(f_{1}, f_{2}, t\right) d f_{1} d f_{2} \\
& \mathrm{LH}(t)=\int_{f_{1}=0^{+}}^{0.15} \int_{f_{2}=0.15^{+}}^{0.4} \mathrm{~B}\left(f_{1}, f_{2}, t\right) d f_{1} d f_{2} \\
& \mathrm{HH}(t)=\int_{f_{1}=0.15^{+}}^{0.4} \int_{f_{2}=0.15^{+}}^{0.4} \mathrm{~B}\left(f_{1}, f_{2}, t\right) d f_{1} d f_{2}
\end{aligned}
$$

\subsection{Statistical analysis}

All HRV features were instantaneously calculated with a $\Delta=5 \mathrm{~ms}$ temporal resolution. In order to build subject-specific feature vectors, for every subject and for evey feature $X$, we condensed the information about the time-varying dynamics of $X$ through its median across time. Given the high variability in individual values of HRV features, median indices during active and sham RAVANS were normalized with respect to the correspondent PB task median values using the:

$$
\begin{aligned}
& \text { individual summary index }= \\
& \quad(\text { median }(P B+R A V A N S)-\text { median }(P B)) / \text { median }(P B)) .
\end{aligned}
$$

Individual values were averaged, one- and two-sample $\mathrm{t}$-tests were used to compare the different conditions. Statistical significance was set at a p-value $<0.05$.

\section{Results}

Point-process goodness-of-fit analyses yielded optimal NARL orders of $\mathrm{p}=4$ and $\mathrm{q}=3$ where, in most recordings, both KS plots and the autocorrelation samples fell within 95\% confidence intervals. Results of group statistics for all features are shown in Table 1. No significant baseline differences in time and frequency domain linear parameters or bispectral features were found between stimulus groups. Normalized values of mean RR interval $\left(\mu_{R R}\right)$ significantly increased during active RAVANS stimulation, both compared to PB alone ( $\mathrm{p}$-value $=0.024)$ and to sham $(\mathrm{p}$-value $=0.043)$. Normalized variations of linear and non-linear vagal indices (HF-HRV and HHHRV) showed a significant increase during Active stimulation when compared to the PB task ( $\mathrm{p}$-value $(\mathrm{HF})=$ 0.048; p-value $(\mathrm{HH})=0.047)$. Furthermore, HF significantly increased during Active sessions compared to Sham sessions $(\mathrm{p}$-value $=0.012$ ), while $\mathrm{HH}$ increase

\begin{tabular}{|c|c|c|}
\hline & SHAM & ACTIVE \\
\hline & $(\mathrm{PB}+\mathrm{RAVANS}-\mathrm{PB}) / \mathrm{PB}$ & $(\mathrm{PB}+\mathrm{RAVANS}-\mathrm{PB}) / \mathrm{PB}$ \\
\hline$\mu_{R R}$ (n.u.) & $0.00 \pm 0.03$ & $0.05 \pm 0.06 *$ \\
\hline$\sigma_{R R}$ (n.u.) & $0.03 \pm 0.50$ & $0.43 \pm 0.75$ \\
\hline LF (n.u.) & $1.16 \pm 2.93$ & $0.18 \pm 0.65$ \\
\hline HF (n.u.) & $-0.25 \pm 0.31$ & $0.47 \pm 0.77 *$ \\
\hline LF/HF(n.u.) & $1.48 \pm 3.75$ & $-0.06 \pm 0.76$ \\
\hline LL (n.u.) & $1.02 \pm 1.24$ & $1.07 \pm 1.46$ \\
\hline LH (n.u.) & $0.22 \pm 0.37$ & $1.02 \pm 1.51$ \\
\hline HH (n.u.) & $0.07 \pm 0.42$ & $1.67 \pm 2.28$ \\
\hline
\end{tabular}
was approaching significance $(\mathrm{p}$-value $=0.056)$ (Figure 1). No significance changes were detected when comparing the Sham sessions to the Paced Breathing (PB) task.

Table 1. Results of Point Process analyses during the sham and active RAVANS sessions. Bold: significant increase during PB+RAVANS compared to PB. *: significant increase during active RAVANS compared to sham.

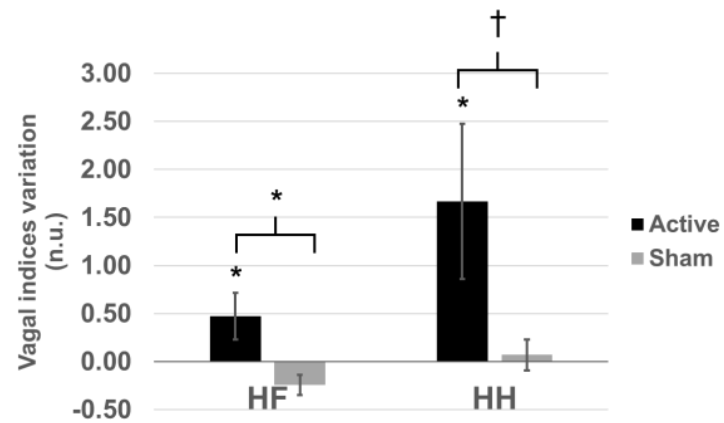

Figure 1 - Normalized variations of linear and non-linear vagal indices (HF-HRV and HH-HRV, respectively). *p-value $<0.05$; $\dagger$ p-value $<0.06$.

\section{Discussion}

Our study reveals that exhalatory-gated afferent vagal stimulation modulates instantaneous complex heart beat dynamics in patients with hypertension.

The instantaneous analysis of bispectral features is a recent development for HRV analysis. It allows the identification of underlying nonlinear interactions occurring between the sympathetic and parasympathetic 
nervous system $[9,10]$. This approach relies on pointprocess models including Wiener-Volterra and Laguerre expansions to calculate reliable estimations of nonlinear heartbeat dynamics [10]. Although the physiological mechanisms behind t-VNS effects on cardiovascular regulation are still unclear, the reported increase in vagal linear and complex dynamics in our study suggests a regulatory action of RAVANS stimulation in the brainstem nuclei and cortical structures involved in autonomic control. This is supported by the fact that inherent complexity and nonstationarity variations of heartbeat dynamics result from nonlinear neural interactions, so that the sinoatrial node responds in a nonlinear manner to the varying levels of efferent autonomic inputs [11]. In the same way as linear sympathetic and parasympathetic effects on HRV are characterized by LF and HF spectral powers [2], it is possible to identify underlying nonlinear sympathovagal interactions by using bispectral analysis in the appropriate frequency bands (LL, HH) [9]. Thus, our findings of an increased $\mathrm{HH}$ in response to RAVANS could be interpreted as possible beneficial effects of this afferent vagal stimulation technique on cardiovascular control functionality of hypertensive patients in terms of a more effective modulation of central efferent parasympathetic outflow. Further studies should be performed to confirm this hypothesis.

The use of a respiratory-gated stimulation could enhance the effects of vagal modulation. This technique is more effective in emulating the natural firing pattern of the vagus nerve by mimicking the breathing-induced modulation of cardiac vagal activity [8]. Previous experimental studies have identified the nucleus tractus solitarii (NTS) as the main structure involved in the processing of vagal afferences [8]. Activation of excitatory $2^{\text {nd }}$-order neurons of the NTS during expiration increases premotor cardiovagal neuron firing rate and inhibits premotor sympathetic neurons $[8,12]$. On the other hand, NTS receives inhibitory inputs from ventral respiratory group (VRG) nuclei in the medulla during inspiration [8]. Our results of enhanced vagal outflow could be explained by increased modulation of NTS by electrical stimulation during exhalation, when this nucleus is not receiving inhibitory influence from VRG, a finding supported by previous neuroimaging studies [13].

\section{Conclusion}

In conclusion, our study is the first to demonstrate beneficial effects of exhalatory-gated vagal stimulation on complex dynamics of parasympathetic activity in hypertensive patients. Future longitudinal studies will be oriented to establish the therapeutic potential of this technique in blood pressure regulation and cardiovascular risk reduction in hypertensive subjects

\section{Acknowledgements}

This study was supported by the Department of Anesthesia, Critical Care and Pain Medicine at Massachusetts General Hospital; the American Heart Association (Grant No. 16GRNT26420084), NIH-Office of Director (OT2-OD023867), and the European Commission LINK project (H2020-692023).

\section{References}

[1] Mancia G, Grassi G. The autonomic nervous system and hypertension. Circ Res. 2014;114(11):1804-14.

[2] Thayer JF, Lane RD. The role of vagal function in the risk for cardiovascular disease and mortality. Biol Psychol. 2007;74(2):224-42

[3] Nearing BD, Libbus I, Amurthur B, Kenknight BH, Verrier RL. Acute Autonomic Engagement Assessed by Heart Rate Dynamics During Vagus Nerve Stimulation in Patients With Heart Failure in the ANTHEM-HF Trial. J Cardiovasc Electrophysiol. 2016;27(9):1072-7.

[4] Daban C, Martinez-Aran A, Cruz N, Vieta E. Safety and efficacy of Vagus Nerve Stimulation in treatment-resistant depression. A systematic review. J Affect Disord. 2008;110(1-2):1-15.

[5] Ellrich J. Transcutaneous Vagus Nerve Stimulation. Eur Neurol Rev. 2011;6(4):254-6.

[6] La Marca R, Nedeljkovic M, Yuan L, Maercker A, Elhert U. Effects of auricular electrical stimulation on vagal activity in healthy men: evidence from a three-armed randomized trial. Clin Sci. 2010;118(8):537-46.

[7] De Couck M, Cserjesi R, Caers R, Zijlstra WP, Widjaja D, Wolf $\mathrm{N}$, et al. Effects of short and prolonged transcutaneous vagus nerve stimulation on heart rate variability in healthy subjects. Auton Neurosci. 2017;203:88-96.

[8] Zoccal DB, Furuya WI, Bassi M, Colombari DSA, Colombari E. The nucleus of the solitary tract and the coordination of respiratory and sympathetic activities. Front Physiol. 2014;5:238.

[9] Valenza G, Citi L, Lanatá A, Scilingo EP, Barbieri R. Revealing real-time emotional responses: a personalized assessment based on heartbeat dynamics. Sci Rep. 2014;4:4998.

[10] Valenza G, Citi L, Scilingo EP, Barbieri R. Point-Process Nonlinear Models With Laguerre and Volterra Expansions: Instantaneous Assessment of Heartbeat Dynamics. IEEE Transactions on Signal Processing. 2013;61(11):2914-26.

[11] Sunagawa K, Kawada T, Nakahara T. Dynamic nonlinear vago-sympathetic interaction in regulating heart rate. Heart and vessels. 1998;13(4):157-74.

[12] Miyazaki M, Tanaka I, Ezure K. Excitatory and inhibitory synaptic inputs shape the discharge pattern of pump neurons of the nucleus tractus solitarii in the rat. Exp Brain Res. 1999;129(2):191-200.

[13] Garcia RG, Lin RL, Lee J, Kim J, Barbieri R, Sclocco R, et al. Modulation of brainstem activity and connectivity by respiratory-gated auricular vagal afferent nerve stimulation in migraine patients. Pain. 2017;158(8):1461-72.

Address for correspondence.

Ronald G. Garcia

CNY 149-2301, $13^{\text {th }}$ St. Charlestown, MA 02129, US.

rgarcia@nmr.mgh.harvard.edu 